\title{
Formación Profesional Basada en Competencias como Estrategia de Apoyo para la Reducción de la Inseguridad Alimentaria en Honduras
}

\author{
Carlos Roberto Ardón ${ }^{1}$ y Ana Afonso Gallegos ${ }^{2}$
}

\begin{abstract}
Resumen. El hambre es un problema que afectaba en el 2011 a 923 millones de personas en el mundo, de las cuales, 52.5 millones estaban en América Latina. En marzo de 2011 el 10.4\% de la población hondureña estaba en situación de inseguridad alimentaria; condición debida básicamente a las condiciones de pobreza y su poder adquisitivo limitado para cubrir la canasta básica de alimentos. La inseguridad alimentaria es un problema complejo y para contrarrestarlo es preciso contar con profesionales con amplio conocimiento y con la capacidad de diseñar políticas y ejecutar programas y/o proyectos que contribuyan a su solución. Con el objetivo de proponer un programa formativo que responda adecuadamente a la demanda de profesionales en Seguridad Alimentaria y Nutricional (SAN), se desarrolló una consulta con informantes claves vinculados a la ejecución de programas y proyectos y a Universidades en Honduras. Los resultados plantean que hay un limitado abordaje de la SAN en la educación superior; situación debida a diversos factores, donde destaca la escasa relación de las universidades con las organizaciones que ejecutan programas y proyectos y por lo tanto un desconocimiento de lo que dichas organizaciones están demandando. Los resultados de la consulta orientan a plantear un modelo de formación denominado Diplomado en Seguridad Alimentaria y Nutricional Basado en Competencias. Este diplomado debería ser el inicio de un proceso de formación que posteriormente evolucione a la implementación de un sistema de certificación de profesionales en seguridad alimentaria y nutricional.
\end{abstract}

Palabras clave: Canasta básica, formación en competencias, seguridad alimentaria.

\section{Competency-based Training as a Support Strategy to Reduce Food Insecurity in Honduras}

\begin{abstract}
Hunger is a problem that affects, in 2011, more than 923 million people worldwide, of which 52.5 million are in Latin America. In March 2011, 10.4\% of the Honduras population was suffering food insecurity, a condition due mainly to poverty and rising food prices. Food insecurity is a complex problem and to reduce it, it's necessary to have professionals with extensive knowledge and the ability to design and implement programs and projects that contribute to its solution. In order to propose a training program that responds adequately to the demand for professionals in Food Security and Nutrition, a consultation was held with key informants associated with the implementation of programs and projects and universities in Honduras. The results indicate that there is a limited approach to food security in higher education, a situation caused by various factors, such as the poor relationship between universities and organizations implementing programs and projects. We propose a Competency-based training in Food Security and Nutritional; this program should be the beginning of a process that progresses to the implementation of a certification system for food security professionals.
\end{abstract}

Key words: Competency-based training, food security.

\section{Introducción}

Según la FAO (1996), hay seguridad alimentaria si "todas las personas tienen en todo momento acceso físico y económico a suficientes alimentos innocuos y nutritivos para satisfacer sus necesidades alimentarias". En 1996 se llevó a cabo en Roma, Italia, la Cumbre Mundial sobre la Alimentación (CMA) y allí se dio inicio una discusión profunda sobre el tema. En dicha cumbre se destacó que 800 millones de personas en todo el mundo padecían de inseguridad alimentaria, definiéndose como el estado en que las

\footnotetext{
${ }^{1}$ Ing. Agrónomo, Máster en Desarrollo Rural. Alumno programa doctorado en Planificación y Gestión de Proyectos de Desarrollo Rural Sostenible. Universidad Politécnica de Madrid. España. Email: cardon682003@yahoo.com

${ }^{2}$ Ing. Agrónomo, Doctora. Docente e investigadora del grupo GESPLAN de la Universidad Politécnica de Madrid. España. Email: ana.afonso@fgupm.es
} 
personas no disponen de suficientes alimentos para satisfacer sus necesidades nutricionales y llevar una vida saludable. Ante tal situación, se planteó entonces el compromiso de reducir a la mitad las personas que padecen hambre y malnutrición el año $2015^{3}$.

En la Cumbre del Milenio 2000 se definieron los Objetivos de Desarrollo del Milenio (ODM $)^{4}$, cuyo objetivo número uno incluye metas específicas en el combate de la pobreza extrema y el hambre. Muchos esfuerzos se han realizado desde entonces, pero los resultados no son los esperados. Existen 923 millones de personas en todo el mundo padeciendo inseguridad alimentaria y 52.5 millones están en América Latina (FAO, 2010). En Honduras, en marzo de 2011, el $10.4 \%$ de la población estaba en situación de inseguridad alimentaria (WFP, 2011). Esta condición es debida básicamente a las condiciones de pobreza y al aumento de precios de la canasta básica, ya que por ello, gran parte de la población, especialmente en las zonas rurales, no puede acceder a los alimentos que necesita. Según PNUD (2010), en 2009 la tasa de pobreza era $58.8 \%$ y la pobreza extrema $36.4 \%$.

La inseguridad alimentaria es un problema complejo y para contrarrestarlo es preciso definir estrategias integrales que aborden la mayoría de factores que la favorecen. Para definir esas estrategias, es necesario contar con profesionales con amplio conocimiento sobre la materia y con la capacidad de traducir dichas estrategias en programas y proyectos en los territorios donde el problema está presente.

La necesidad de formación de profesionales en

\footnotetext{
3 «Prometemos consagrar nuestra voluntad política y nuestra dedicación común y nacional a conseguir la seguridad alimentaria para todos y a realizar un esfuerzo constante para erradicar el hambre de todos los países, con el objetivo inmediato de reducir el número de personas desnutridas a la mitad de su nivel actual no más tarde del año 2015.» (Declaración de Roma, 1996).

4 En la asamblea general de Naciones Unidas en septiembre del 2000 se definieron y aprobaron ocho objetivos de desarrollo que contienen 21 metas a lograr en el 2015. Estos objetivos tienen relación con la pobreza, educación, salud y medio ambiente. http://www.undp.org/spanish/mdg/
}

Seguridad Alimentaria y Nutricional (SAN) no es algo nuevo en la región. En el 2004, el Consejo Superior Universitario de Centroamérica (CSUCA) elaboró y aprobó el Plan de Acción Universitario para la Promoción de la Seguridad Alimentaria y Nutricional (PAUSAN), un plan con el objetivo de sensibilizar a la comunidad universitaria Centroamericana para incluir la SAN en la formación, investigación y extensión en cada centro universitario (CSUCA et al. 2004). Los avances en el plan no han sido los esperados, aunque se ha capacitado personal técnico de gobiernos, ONG y de las universidades, no se ha avanzado lo suficiente en la creación de programas permanentes de formación en SAN o en la inclusión del tema en las universidades. En el cuadro 1 se describen los programas especializados en SAN más representativos en Centroamerica. Una característica común es que han sido creados con el apoyo de la cooperación internacional, donde destaca la FAO a través de sus programas y proyectos en la región.

A pesar de que la inseguridad alimentaria es uno de los principales problemas en la región, el involucramiento de las universidades en procesos de formación e investigación es limitado. Este aspecto ha sido ampliamente estudiado en América Latina, donde varios autores han destacado ese débil vínculo entre la educación superior (universidades) y los procesos de investigación y extensión, contrario a los países desarrollados donde la universidad es un actor determinante en los procesos de desarrollo (Plata, 2003; Carrión, 2003). Lo que sucede en los países latinoamericanos no es responsabilidad exclusiva de las universidades, ya que el apoyo que éstas reciben también es limitado. Un ejemplo de eso es el gasto en investigación y desarrollo (\% del PIB) publicado por el Banco Mundial ${ }^{5}$, donde Estados Unidos y Alemania aparecen con los indicadores más altos (más de 2.5\%) y la mayoría de países latinoamericanos dedican menos del $0.5 \%$ en generar nuevos conocimientos para beneficio de la sociedad.

\footnotetext{
${ }^{5}$ Disponible en http://datos.bancomundial.org/indicador/GB.XPD.RSDV. GD.ZS, consultado el 11 enero de 2012.
} 
Cuadro 1. Principales programas especializados en Seguridad Alimentaria y Nutricional (SAN) en Centroamérica.

\begin{tabular}{|c|c|c|}
\hline País & Programa & Instituciones involucradas \\
\hline Nicaragua & $\begin{array}{l}\text { Posgrado en Pobreza y SAN en } \\
\text { Universidades de Nicaragua }^{1}\end{array}$ & $\begin{array}{l}\text { Universidad Nacional Autónoma de Nicaragua-León, } \\
\text { Universidad Centroamericana (UCA) y Universidad } \\
\text { Nacional Agraria (UNA). }\end{array}$ \\
\hline Costa Rica & Maestría en Geren & $\begin{array}{l}\text { Escuela de Ciencias Agrarias de la Universidad } \\
\text { Nacional de Costa Rica y la Escuela de Nutrición de } \\
\text { la Universidad de Costa Rica. }\end{array}$ \\
\hline Guatemala & $\begin{array}{l}\text { Posgrados en Pobreza y Seguridad } \\
\text { Alimentaria }{ }^{3} \\
\text { Maestría en Alimentación y Nutrición } \\
\text { (MANA) }\end{array}$ & $\begin{array}{l}\text { Programa PESA, Facultad Latinoamericana de } \\
\text { Ciencias Sociales (FLACSO), Universidad de San } \\
\text { Carlos y Universidad Rafael Landivar. } \\
\text { Facultad de Química y Farmacia de la Universidad } \\
\text { San Carlos (USAC) }\end{array}$ \\
\hline Honduras & Profesorado en SAN & $\begin{array}{llll}\begin{array}{l}\text { Universidad } \\
\text { Morazán }\end{array} & \text { Pedagógica } & \text { Nacional } & \text { Francisco } \\
\end{array}$ \\
\hline $\begin{array}{l}\text { Otras } \\
\text { experiencias }\end{array}$ & $\begin{array}{l}\text { Cursos FODEPAL } \\
\text { Maestría en Seguridad Alimentaria y } \\
\text { Nutricional (SAN) } \\
\text { REDSAN }\end{array}$ & $\begin{array}{l}\text { Experiencias de formación en SAN por medio de } \\
\text { cursos online. } \\
\text { PRESANCA II, PRESISAN, USAC, UNAN } \\
\text { Managua, UNAN León, Universidad de Panamá. } \\
\text { Red de investigación en seguridad alimentaria y } \\
\text { nutricional. Escuela de Nutrición, Universidad de } \\
\text { Costa Rica. }\end{array}$ \\
\hline
\end{tabular}

Fuente: ${ }^{1}$ UNAN-León; UCA y UNA, 2006; ${ }^{2}$ UCR y UNCR, 2009; ${ }^{3}$ Barillas, 2004 y FAO, et al. 2006; www.usac.edu.gt/postgrados.php

Otro elemento determinante en la participación de las universidades en el desarrollo de la sociedad es la innovación, un aspecto muy relacionado con la investigación. La innovación es el proceso mediante el cual se convierte el conocimiento en Producto Interior Bruto y Bienestar, mediante la creación de nuevos productos o servicios o la mejora de los existentes. En cierto sentido la innovación es el proceso inverso a la investigación; investigar es extraer conocimiento del estudio de la realidad e innovar es aplicar conocimiento con el objetivo de conformar una nueva realidad. Para que se produzca la innovación se requiere un continuo Proceso de Aprendizaje (Nonaka y Takeuchi, 1995)

Siendo la innovación un fin último de la investigación, se presenta como una tercera función de la universidad. La misión de la universidad ya no es solamente formar profesionales y generar conocimiento a través de la investigación, sino que también tiene una función de transferencia de conocimiento y cada día mayor compromiso de participación activa en la generación de riqueza en la sociedad a la cual sirve (Juliá, 2007). Esta responsabilidad de asumir un rol determinante en los procesos innovadores que promueven el desarrollo, no es algo alejado de las otras funciones de la universidad, al contrario, es pertinente a la misión primaria tradicional de la universidad de investigación y formación (Lester, 2005).

\section{Antecedentes y Objetivos}

Ardón y Afonso (2009) estudiaron las percepciones en Honduras para conocer la oferta y demanda de formación e investigación en SAN, así como la percepción de la participación de las 
universidades en la lucha contra el hambre en el país. El estudio se llevó a cabo por medio de una encuesta dirigida a profesionales vinculados a la SAN del sector público y privado. Uno de los resultados del estudio fue que las personas encuestadas perciben que la educación superior poco contribuye a la solución de las limitantes que condicionan la inseguridad alimentaria. Esta posición es explicada en parte por la limitada oferta académica en el tema y la escasa participación de las universidades en los foros de discusión sobre la SAN en el país. Dicho estudio también determinó que para fortalecer las capacidades profesionales en SAN se requieren programas que brinden una formación integral que permitan el desarrollo no solo de competencias técnicas, sino también de las competencias de comportamiento requeridas en los profesionales para relacionarse con los todos los actores; también son importantes las competencias contextuales, que son básicas para entender el entorno.

La principal conclusión de dicho estudio fue que las universidades en Honduras tienen en la SAN un espacio propicio para cumplir su función (formación, investigación/innovación y extensión) y que existe una demanda no satisfecha de formación e investigación en SAN que debe ser más estudiada.

En seguimiento a ese estudio, y con el objetivo general de proponer un programa formativo que responda adecuadamente a la demanda de profesionales en SAN, se consultaron informantes claves vinculados a la ejecución de programas y proyectos y a Universidades en Honduras.

Para lograr el objetivo general, se plantearon los siguientes objetivos específicos:

- Determinar el nivel de abordaje de la SAN en la educación superior.

- Identificar el tipo de profesionales que contratan las organizaciones que ejecutan programas y proyectos; y las capacidades en SAN que éstos poseen.

- Definir la modalidad de formación más aceptada y los contenidos académicos que debería tener un programa de formación en SAN.

- Explorar el interés en un proceso de formación y certificación en competencias en SAN.

\section{Metodología}

La consulta fue desarrollada mediante entrevistas semiestructuradas aplicadas a una muestra de expertos vinculados a programas y proyectos de seguridad alimentaria y a profesionales que laboran en las principales universidades en Honduras. La entrevista incluyó preguntas cerradas donde el entrevistado debía seleccionar una respuesta entre determinadas opciones y también preguntas abiertas, donde cada uno tenía la oportunidad de expresar su opinión sobre los temas de la consulta.

La entrevista a profesionales de programas y proyectos tuvo por objetivo profundizar en la demanda de formación en SAN en las organizaciones; la entrevista a profesionales de universidades permitió conocer la oferta de formación en SAN en los programas académicos y el interés de los centros universitarios para involucrarse en acciones que conduzcan a satisfacer la demanda existente. En total se entrevistaron 25 personas distribuidas de la siguiente manera: 10 de organizaciones de desarrollo, 12 de instituciones universitarias y 3 de la cooperación internacional. Los miembros de organizaciones de desarrollo y cooperación internacional fueron seleccionados de una lista de participantes del Comité Técnico Interinstitucional en Seguridad Alimentaria y Nutricional (COTISAN) ${ }^{6}$. En la selección de miembros de universidades se consideró el criterio que las mismas contaran con facultades o programas vinculados al desarrollo, especialmente del sector rural, y se priorizó entrevistar a docentes o directivos de dichas facultades. Otro criterio de selección fue la disponibilidad de los profesionales para entrevistarlos en sus lugares de trabajo.

El proceso de recolección y análisis de información se muestra en la figura 1. Consistió en recopilar la información por medio de la entrevista, organizar dicha información, transcribir la información en un procesador de texto, revisión de la misma para asegurar que toda la información

${ }^{6}$ COTISAN es un grupo interdisciplinario que actúa como una instancia de consulta y asistencia coordinado por la Unidad Técnica de Seguridad Alimentaria y Nutricional (UTSAN) 
importante estuviese incluida, agrupar la información de acuerdo a los objetivos planteados en el estudio, revisión detallada de la información, extracción de aspectos relevantes y generación de conclusiones alrededor de la misma.

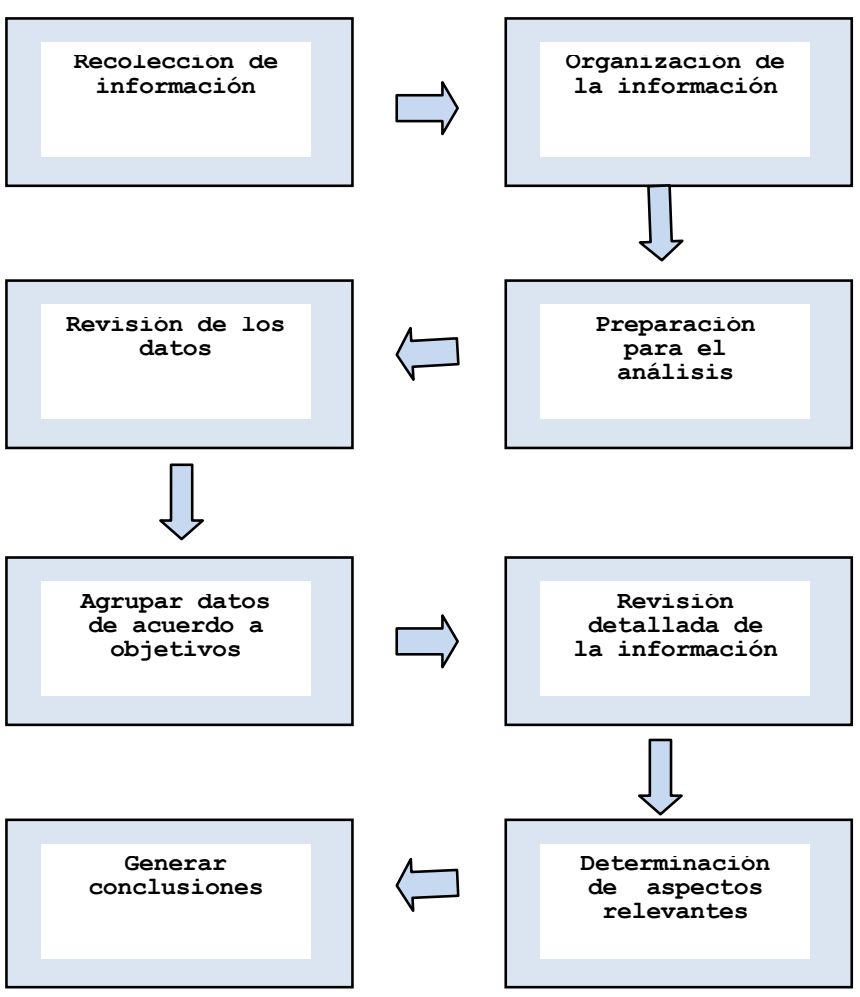

Figura 1. Proceso de recolección y análisis de información.

Además de la recolección de información primaria por medio de la entrevista, también se realizó una revisión de información bibliográfica sobre los temas abordados. Dicha información se presenta de manera conjunta con los resultados de la entrevista y su objetivo es profundizar sobre los elementos clave surgidos en la investigación.

\section{Resultados y Discusión}

Nivel de abordaje de la SAN en la educación superior en Honduras. La máxima autoridad de la educación superior en Honduras es La Universidad Nacional Autónoma de Hondura (UNAH), la cual es responsable de organizar y dirigir la educación superior por medio de los siguientes órganos: Consejo Universitario, Consejo de Educación Superior, Consejo Técnico Consultivo y Dirección de Educación Superior. Estas instancias son las responsables de aprobar los estudios universitarios en el país, ya sean por medio de nuevos centros universitarios o nuevos programas académicos promovidos por los centros ya establecidos. En el cuadro 2 se listan las instituciones de educación superior registradas al año 2008 en Honduras.

La mayoría de universidades cuentan con programas de pregrado y posgrados, mostrando los últimos años un avance interesante en la oferta de posgrados en diferentes áreas temáticas. Quiñones y Vélez (2004) destacan que aunque ha aumentado la oferta de posgrados, la función de investigación y transferencia de conocimientos y/o tecnología es la menos desarrollada en las universidades Latinoamericanas y constituye una seria crítica por los estudiosos del tema.

En relación a la generación de información por medio de la investigación, Oseguera (2005) determinó que de 16 universidades que existían en el 2004 en Honduras, solo cuatro de ellas contaban con políticas de investigación y con mecanismos de apoyo a los docentes para la labor investigativa y para publicar sus resultados. Esta investigación se ha concentrado en las áreas de la salud, agricultura y educación. Esta realidad indica que todavía falta mucho por hacer, para que la academia desarrolle su labor como motor de cambio en la sociedad actual.

En cuanto al abordaje de la SAN, en 2011, el único programa específico en SAN era el profesorado en SAN ofertado por la Universidad Pedagógica Nacional Francisco Morazán (UPNFM). Es un programa de pregrado cuyo objetivo es formar docentes de educación media en SAN con el grado académico de Licenciatura. Su plan de estudios se enfoca en tecnología de alimentos, con elementos muy marcados en calidad, higiene y manipulación de los mismos ${ }^{7}$.

${ }^{7}$ Información revisada en: www.upnfm.edu.hn 
Cuadro 2. Centros de educación superior registrados en Honduras en el 2008.

\begin{tabular}{lll}
\hline Nombre del centro de educación superior & Siglas & Naturaleza \\
\hline Universidad Nacional Autónoma de Honduras & UNAH & Estatal \\
Universidad José Cecilio del Valle & UJCV & Privada \\
Universidad de San Pedro Sula & USPS & Privada \\
Universidad Tecnológica Centroamericana & UNITEC & Privada \\
Seminario Mayor Nuestra Señora de Suyapa & SMNSS & Privada \\
Universidad Pedagógica Nacional Francisco Morazán & UPNFM & Estatal \\
Universidad Tecnológica de Honduras & UTH & Privada \\
Escuela Agrícola Panamericana & EAP & Privada \\
Universidad Nacional de Agricultura & UNAG & Estatal \\
Escuela Nacional de Ciencias Forestales & ESNACIFOR & Estatal \\
Universidad Católica de Honduras Nuestra Señora Reina de la Paz & UNICAH & Privada \\
Centro de Diseño, Arquitectura y Construcción & CEDAC & Privada \\
Instituto Superior de Educación Policial & ISEP & Estatal \\
Universidad Cristiana Evangélica Nuevo Milenio & UCENM & Privada \\
Universidad Metropolitana de Honduras. & UMH & Privada \\
Universidad Cristiana de Honduras & UCRISH & Privada \\
Instituto Tecnológico Superior Jesús de Nazareth & ITSJN & Privada \\
Universidad Politécnica de Honduras & UPH & Privada \\
Universidad de Defensa de Honduras & UDH & Estatal \\
Universidad Politécnica de Ingeniería de Honduras & UPI & Privada \\
\hline
\end{tabular}

Fuente: Registro de Universidades de la Dirección de Educación Superior en Honduras (UNAH, 2008)

En el 2011, la Universidad Nacional Autónoma de Honduras (UNAH) estuvo diseñando una carrera de nutrición a nivel de licenciatura y una Maestría en Seguridad Alimentaria y Nutricional. También existen planes de conformar un sistema nacional de investigación en SAN en donde puedan participar los posgrados que el centro universitario ofrece ${ }^{8}$.

Aunque la oferta de formación específica en SAN es limitada, hay programas académicos relacionados con SAN, ya sea desde el punto de vista de producción y procesamiento de alimentos, como de la nutrición y la salud humana. La mayoría de ellos son ofertados por la Universidad Nacional Autónoma de Honduras (UNAH) y en su mayoría (85\%) son programas de pregrado. En el cuadro 3 se listan los programas académicos más representativos vinculados con la SAN existentes en la educación superior en Honduras.

Todas las personas entrevistadas consideran que

\footnotetext{
${ }^{8}$ Comunicación personal con Dra. Olga Joya, Directora de Posgrados de la UNAH (mayo, 2011).
}

la SAN tiene un limitado abordaje en la educación superior y que la razón principal es la falta de profesionales con formación en SAN en las universidades. Indican que la materia se trata como parte de asignaturas más generales y en seminarios complementarios, pero se requiere un abordaje más especializado. Ambas respuestas están relacionadas, ya que sin profesionales capacitados no es posible incluir en los currículos académicos los temas de la SAN; por tal razón es imperativo que el país considere la formación superior en SAN como prioridad.

Un aspecto a destacar en relación al abordaje de la SAN en los centros de educación superior y en la sociedad hondureña en general, es que fue hasta en los inicios de la década del 2000 que en el país se inicio un abordaje mas integral de la problemática; un enfoque que en otras latitudes estaba siendo abordado mucho más antes 9 .

${ }^{9}$ Comunicación personal Ing. Milton Flores. Representante Asistente FAO Honduras. 10 enero de 2012 
Cuadro 3. Programas relacionados con la Seguridad Alimentaria y Nutricional (SAN) ofertados por las universidades en Honduras

\begin{tabular}{|c|c|c|}
\hline Universidad & Programa & Grado académico \\
\hline $\begin{array}{l}\text { Universidad } \\
\text { Nacional Autónoma } \\
\text { de Honduras } \\
\text { (UNAH) }\end{array}$ & $\begin{array}{l}\text { Ciencias Acuícolas } \\
\text { Procesamiento de Carnes } \\
\text { Procesamiento de Lácteos } \\
\text { Procesamiento de Granos y Semillas } \\
\text { Producción Agrícola } \\
\text { Producción Pecuaria } \\
\text { Tecnología de Alimentos } \\
\text { Educación para la Gestión y Desarrollo local } \\
\text { Administración de Empresas Agropecuarias } \\
\text { Ingeniería Agroindustrial } \\
\text { Ingeniería Agronómica } \\
\text { Ingeniería en Ciencias Acuícolas y Recursos Marino Costeros } \\
\text { Trabajo Social } \\
\text { Medicina } \\
\text { Enfermería } \\
\text { Trabajo Social con Énfasis en Gestión del Desarrollo } \\
\text { Planificación y Desarrollo } \\
\text { Ciencias Sociales con Énfasis en Gestión del Desarrollo }\end{array}$ & $\begin{array}{l}\text { Técnico universitario } \\
\text { Técnico universitario } \\
\text { Técnico universitario } \\
\text { Técnico universitario } \\
\text { Técnico universitario } \\
\text { Técnico universitario } \\
\text { Técnico universitario } \\
\text { Técnico universitario } \\
\text { Licenciatura } \\
\text { Licenciatura } \\
\text { Licenciatura } \\
\text { Licenciatura } \\
\text { Licenciatura } \\
\text { Licenciatura } \\
\text { Licenciatura } \\
\text { Maestría } \\
\text { Maestría } \\
\text { Doctorado }\end{array}$ \\
\hline $\begin{array}{l}\text { Escuela Agrícola } \\
\text { Panamericana }\end{array}$ & $\begin{array}{l}\text { Ciencia y Producción Agropecuaria } \\
\text { Agroindustria Alimentaria } \\
\text { Desarrollo Socioeconómico y Ambiente }\end{array}$ & $\begin{array}{l}\text { Licenciatura } \\
\text { Licenciatura } \\
\text { Licenciatura }\end{array}$ \\
\hline $\begin{array}{l}\text { Universidad } \\
\text { Nacional de } \\
\text { Agricultura }\end{array}$ & $\begin{array}{l}\text { Ingeniería Agronómica } \\
\text { Tecnología Alimentaria }\end{array}$ & $\begin{array}{l}\text { Licenciatura } \\
\text { Licenciatura }\end{array}$ \\
\hline $\begin{array}{l}\text { Universidad } \\
\text { Pedagógica Nacional } \\
\text { Francisco Morazán }\end{array}$ & Profesorado en Seguridad Alimentaria y Nutricional & Licenciatura \\
\hline $\begin{array}{l}\text { Universidad } \\
\text { Tecnológica } \\
\text { Centroamericana } \\
\text { (UNITEC) }\end{array}$ & Maestría en desarrollo local y turismo & Maestría \\
\hline $\begin{array}{l}\text { Universidad } \\
\text { Cristiana Evangélica } \\
\text { Nuevo Milenio }\end{array}$ & Salud Comunitaria & Licenciatura \\
\hline
\end{tabular}

Tipo de profesionales que trabajan en contratan profesionales universitarios que cursan por programas y proyectos de apoyo a la SAN y lo general cuatro años de educación superior formación de los mismos. Las organizaciones que (ingenieros y licenciados) y técnicos de nivel medio. ejecutan programas y proyectos vinculados a la SAN Las entrevistas realizadas, han permitido determinar 
que las principales áreas de formación de dichos contratados son: agronomía $(60 \%)$, trabajo social (15\%), nutrición, enfermería y medicina (25\%). También se determinó que la proporción de profesionales de las diferentes áreas depende de la naturaleza de la organización. Si los programas y proyectos están encaminados a incrementar la producción agrícola y mejorar el acceso a mercados, predominan los agrónomos. Si el enfoque de las acciones es nutrición y salud familiar, la mayoría de profesionales son trabajadores sociales y profesionales de la medicina.

El 84\% de las personas entrevistadas que laboran en ONG, argumentaron que los profesionales que contratan están entre mal y regularmente capacitados en SAN y que para actualizar los conocimientos de su personal, deben desarrollar periódicamente eventos de capacitación, donde brindan los conceptos básicos sobre el tema, la situación actual a nivel de país y las metodologías de abordaje que desarrolla cada organización. Solo el $16 \%$ argumentó que los profesionales están entre bien y excelentemente capacitados en el tema SAN.

Ante la pregunta de cuáles son las principales limitaciones que presentan los egresados universitarios, predomina la opinión que los profesionales manejan en buena medida los aspectos relacionados con su especialidad, pero hace falta un conocimiento más integral sobre los aspectos que condicionan la inseguridad alimentaria en el país y las estrategias de solución. Esta limitante debe ser subsanada porque para desempeñarse eficientemente en un programa o proyecto, los profesionales deben conocer en detalle los pilares de la SAN (disponibilidad, acceso, consumo y estabilidad de los alimentos), así como los factores que influyen sobre cada uno de ellos. Las personas consultadas a nivel de organizaciones de desarrollo, atribuyen esa escasa formación en el tema SAN de los egresados universitarios, a la casi inexistente interacción entre las universidades y las organizaciones (públicas y privadas) receptoras de sus egresados. Se plantea como una necesidad, la creación de espacios de diálogo (foros) donde ambos actores puedan interrelacionarse y donde las universidades accedan a información de parte de la demanda, que les permita avanzar hacia programas de formación más pertinentes con la realidad o con lo que la sociedad realmente necesita. Esta realidad es un ejemplo de lo que sucede en otros ámbitos dentro de Honduras, donde la falta de coordinación e integración de acciones entre instancias es un factor común.

Modalidad de formación y contenidos académicos recomendados para programas de formación en SAN. La SAN incluye una temática muy diversa y tal como lo expresa su concepto, tiene relación con la producción de alimentos inocuos y nutritivos, nivel de acceso a esos alimentos por parte de la población, formas de consumo de dichos alimentos y su impacto en la nutrición de las personas. Debido a esa diversidad temática, en los programas y proyectos que desarrollan acciones para mejorar la SAN, se requiere la participación de equipos multidisciplinares, que de manera coordinada ejecuten acciones en un territorio definido para lograr mejorar la calidad de vida de una población específica. Considerando esa diversidad de conocimientos requeridos. El $72 \%$ de las personas consultadas considera que para fortalecer las capacidades profesionales en SAN en Honduras es pertinente la creación de modelos de formación continua (estudios complementarios) para los profesionales que ya están involucrados en programas y proyectos. Un $16 \%$ considera que la SAN debe ser una disciplina transversal en la educación superior y un $12 \%$ opina que es preciso implementar programas específicos de formación en SAN.

Predomina la percepción que no solo a nivel superior es necesario transmitir conocimientos sobre la SAN, sino que también a nivel de la educación primaria y media se debería brindar formación encaminada a modificar los hábitos alimenticios de los estudiantes, para mejorar así, su estado nutricional. En Centroamérica los PESA ${ }^{10}$ han desarrollado interesantes experiencias en este sentido, donde por medio de programas de huertos escolares, alimentación escolar y capacitación de la comunidad educativa (maestro, alumno y padres de familia) han logrado cambios importantes en la alimentación y nutrición de los escolares. Programas de este tipo son muy

${ }^{10}$ http://www.pesacentroamerica.org/index.php 
importantes, ya que los niños y jóvenes son una excelente vía para transmitir conocimientos y cambios de comportamiento a sus familias y entorno más inmediato.

Para conocer la preferencia sobre los contenidos de los programas de formación, en el proceso de consulta se proporcionó a los encuestados una serie de contenidos (Cuadro 4) y se solicitó asignar a los mismos un grado de importancia mediante la siguiente escala cualitativa: $1=$ extremadamente importante, $2=$ muy importante, $3=$ bastante importante y $4=$ poco importante. Para calcular el grado de importancia combinado, el número de respuestas en cada uno de los contenidos, se multiplicó por un valor asignado a cada elemento de la escala cualitativa y luego se sumaron los resultados obtenidos. El resultado del cálculo es una comparación entre grado de importancia (mayor a menor) percibido sobre los diferentes contenidos. Los valores utilizados para calcular el grado de importancia se detallan en el cuadro 5 y los resultados se presentan en la figura 2.

Cuadro 4. Contenidos propuestos para programas de formación en Seguridad Alimentaria y Nutricional (SAN)

\section{Tema Principales elementos que incluye}

Conceptos generales sobre pobreza, Conceptos, medición, tipos de hambre, pilares de la SAN, desarrollo humano y SAN Soberanía Alimentaria, cambio climático.

Causas y situación actual a nivel Causas, situación a nivel mundial y América Latina, proyecciones mundial de organismos internacionales.

Situación en Honduras

Impacto del hambre en el desarrollo

Propuestas de solución a la situación actual

Institucionalidad y políticas de apoyo a la SAN

Planificación de proyectos de apoyo a la SAN

Seguimiento y monitoreo de acciones en SAN

Educación para mejorar la SAN
Análisis detallado de los pilares de la SAN: disponibilidad, acceso, consumo y estabilidad de los alimentos.

Costo del hambre, relación entre nutrición y desarrollo, vulnerabilidad a enfermedades, nutrición y educación.

Cumbres internacionales, propuestas de solución, programas de apoyo a nivel regional y local, experiencias exitosas, rol de la pequeña agricultura.

Grupos de trabajo a nivel mundial, institucionalidad de apoyo a la SAN en Honduras, marco de políticas, rol de los distintos actores (gobierno, ONG, Sociedad Civil, Cooperación internacional, sector privado)

Definición de territorios prioritarios, acciones en caso de emergencias alimentarias, planificación de largo plazo.

Definición de indicadores y medición de los mismos

Hábitos alimenticios de la población, calidad e inocuidad de alimentos, educación en el hogar, acciones en centros educativos, rol de los medios de comunicación. 
Cuadro 5. Escala usada para valorar la importancia de los contenidos de los programas de formación.

\begin{tabular}{lc}
\hline Escala cualitativa & Valor \\
\hline 1 Extremadamente importante & 1.00 \\
2 Muy importante & 0.75 \\
3 Bastante importante & 0.50 \\
4 Poco importante & 0.25 \\
\hline
\end{tabular}

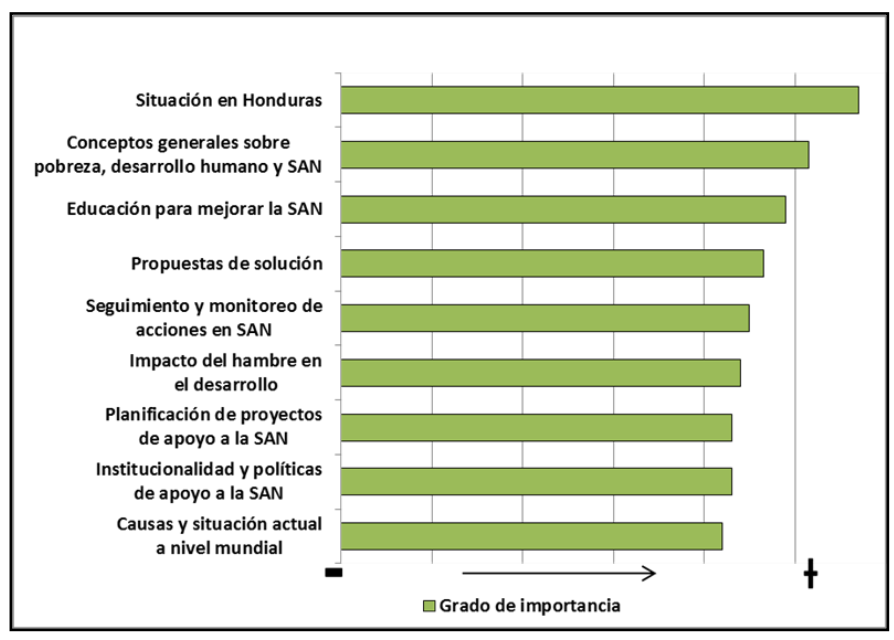

Figura 2. Grado de importancia asignado a contenidos relacionados con la Seguridad Alimentaria y Nutricional.

Todos los contenidos son valorados con un nivel de importancia alto. Además, todos los encuestados creen que dichos contenidos deben ser abordados en las carreras específicas en SAN y en las carreras relacionadas, destacando que en caso de una especialización, los mismos se deben abordar con la mayor profundidad posible. Las respuestas son lógicas, ya que, por lo general, a las personas les interesa primero conocer la situación de su entorno más inmediato y después lo que pasa a nivel global. En el caso de la SAN, en varias ocasiones las causas no son necesariamente locales, así que el conocimiento de lo que pasa a nivel global es tan importante como la situación local. Los contenidos considerados en la entrevista son los que normalmente están considerando los programas académicos establecidos en otros países y se puede asegurar que engloban la mayoría de la temática relacionada; prueba de ello es que durante la entrevista no hubo sugerencias sobre la inclusión de otros temas.

\section{Formación en competencias y certificación de profesionales en SAN}

El término competencia tiene múltiples definiciones. El psicólogo David McClelland, de la Universidad de Harvard, lo definió como "El conjunto de conocimientos, actitudes y destrezas necesarias para desempeñar una ocupación dada" (Brígido, 2008).

Según Serón (1999), que cita a Bunk 1994, competencia es "La capacitación real para resolver determinados problemas, contando además de los conocimientos, destrezas y aptitudes, o la flexibilidad y autonomía, con las competencias "humanas y sociopolíticas", que amplían el radio de acción a la participación en el entorno profesional, así como a la organización del trabajo y a las actividades de planificación"

Tobon (2008), define la competencia como "Procesos complejos de desempeño con idoneidad en determinados contextos, integrando diferentes saberes (saber ser, saber hacer, saber conocer y saber convivir), para realizar actividades $y / 0$ resolver problemas con sentido de reto, motivación, flexibilidad, creatividad, comprensión y emprendimiento, dentro de una perspectiva de mejoramiento continuo y compromiso ético"

Otra definición bastante aceptada es la que propone AEIPRO e IPMA (2006) cuando dice que "competencia es un compendio de conocimiento, actitud personal, destrezas y experiencia relevante, necesario para tener éxito en una determinada función"

Las definiciones anteriores permiten entender que el término competencia se refiere a la capacidad que tiene la persona para desarrollar una determinada función y alcanzar los objetivos propuestos y que, para obtener esa capacidad, no solo es necesario poseer conocimientos sobre la tarea a desempeñar, sino que también es importante la actitud personal, las relaciones con los demás y el conocimiento del entorno. 
El estudio de las competencias no es nuevo. Sin embargo, según Arguelles y Gonczi (2001), su manifestación más reciente data de unos 15 años y surgió por un interés económico, para adecuar la educación y capacitación vocacional a las necesidades de la industria del momento. Dicha formación estuvo concebida para lograr mano de obra calificada y volver más competitivo al sector industrial.

A finales de la década de los setenta, y como producto de las transformaciones socioeconómicas de la época, se inicia el debate sobre la formación profesional basada en competencias (Rodríguez, 2009). Dicho debate tenía por objetivo impulsar formas novedosas de formación profesional de acuerdo a lo que el mercado laboral estaba necesitando. Más adelante, y producto del fenómeno de la globalización y la necesidad de competir en los mercados internacionales, en los años 80 surgió en Estados Unidos un movimiento para acercar la enseñanza en la universidad al puesto de trabajo, movimiento que posteriormente se extendió a otras regiones del mundo y fue el origen de la concepción moderna de la Educación Basada en Normas de Competencia (EBNC). La EBNC, es por tanto, el proceso de adaptar la educación y formación de personas a las necesidades y demandas de la sociedad.

Ardón y Afonso (2009) determinaron que en Honduras los profesionales vinculados a programas y proyectos de apoyo a la SAN le asignan una importancia primaria a la necesidad de implementar programas de formación basados en competencias. También determinaron que los programas de formación deben ser integrales y procurar no solo la transmisión de conocimientos técnicos, sino también competencias que les permita a los egresados entender las variables del entorno y relacionarse de manera eficiente con los diversos actores involucrados en un determinado territorio o campo de acción.

En este estudio se consultó a los entrevistados su opinión sobre la factibilidad de implementar un programa de formación y certificación de profesionales en SAN, la utilidad del mismo y quien debería liderarlo. Todas las personas entrevistadas manifestaron que sería importante la implementación de procesos de formación basados en competencias, para analizar la posibilidad de un proceso de certificación de profesionales. El argumento más generalizado es que un proceso de este tipo per mitiría a las organizaciones contar con el personal idóneo para sus programas y proyectos; además, los profesionales serían más competitivos y contribuirian más al desarrollo del país. A la pregunta sobre quien debería liderar un proceso de esta naturaleza, hay consenso que el mismo debe ser liderado por alguna universidad que tenga experiencia en procesos de este tipo o que esté interesada en generar una experiencia piloto que sea la base de iniciativas más amplias.

\section{Propuesta de Acciones}

Los resultados de la consulta son la base para plantear un modelo de formación denominado Diplomado en Seguridad Alimentaria y Nutricional Basado en Competencias. Un diplomado es un tipo de formación aprobado por el consejo de educación superior, cuyo objetivo es la transmisión de conocimientos y el desarrollo de habilidades en un tema puntual y que responde a una demanda específica. Los diplomados van dirigidos a profesionales que ya se desempeñan en alguna función y que están interesados en actualizar y mejorar sus conocimientos en su campo de acción. Los diplomados generalmente están divididos en módulos que se brindan por separado y que de manera conjunta constituyen la base de conocimientos que se requiere fortalecer.

El programa de formación propuesto deberá cumplir con una serie de características y requisitos que se resumen a continuación.

Objetivo y público meta: el objetivo es fortalecer las capacidades profesionales en SAN como estrategia para reducir la inseguridad alimentaria en Honduras, que favorezcan una mejora de las intervenciones que desarrollan instituciones del sector público y privado. El diplomado debe estar dirigido a profesionales vinculados a proyectos y programas de apoyo a la SAN, que estén interesados en actualizar y mejorar sus conocimientos en torno al tema. También podrán acceder profesores del nivel medio o superior que quieran ampliar sus conocimientos para luego transmitirlos a sus estudiantes. 


\section{Modelo de formación basado en competencias}

De acuerdo a Mertens (2000) existen tres modelos por los que se puede originar e implementar un sistema de formación en competencias. Uno de ellos es cuando el proceso es ideado e impulsado por el sistema educativo de un país o región. Otra forma es cuando el proceso es apoyado y dirigido coordinadamente entre los agentes sociales (gobierno, sociedad civil y sector privado). Este modelo se ha promovido en países donde el sistema educativo había quedado rezagado y no satisfacía las demandas laborales de la sociedad. El tercer modelo es una mezcla, en la que el sistema educativo promueve la formación en competencias junto con los actores sociales, pero a la vez integrándolo en las políticas regionales o nacionales de mercado de trabajo, es decir, no se queda en simple formación profesional sino que avanza al establecimiento de normas de contratación de personal por parte del sector publico y privado para los egresados del sistema.

Brigido (2008) argumenta que la certificación en competencias es importante porque garantiza la autenticidad y la capacidad profesional de las personas certificadas. Admite también que la certificación ha adquirido mayor relevancia porque los programas tradicionales se vuelven obsoletos y son superados por las transformaciones tecnológicas; por tanto la certificación garantiza la actualización de los profesionales y un buen servicio a los demandantes de empleo. Según Guerrero et al. (2010), una característica fundamental de una certificación es que la formación está desvinculada de la certificación misma, la cual es responsabilidad de un organismo especializado que debe regirse por normas $y$ estándares internacionales. La norma internacional en certificación de personas es la ISO/IEC 17024, la cual establece los requisitos que deben cumplir los organismos de certificación para lograr coherencia, comparabilidad y confiabilidad en el proceso de certificación. La norma ISO/IEC 17024 es una guía; cada organismo de certificación podrá complementarla considerando las necesidades específicas del mercado o requisitos gubernamentales (AENOR, 2003).

En el Diplomado en SAN, la formación en competencias será transversal en todos los módulos y el objetivo es generar una primera experiencia de aprendizaje que sea la base para la implementación de un programa de certificación de profesionales en el tema. Para esto, es preciso identificar en Honduras la institución o instituciones que en el futuro podrían liderar un proceso de este tipo. Las competencias a fortalecer en cada uno de los módulos se presentan en el cuadro 6.

Como es evidente, el Diplomado en SAN fortalecerá en los participantes competencias de orden técnico, contextuales y de comportamiento; de tal manera que al final del proceso los participantes del mismo sean capaces de mejorar sus intervenciones y la de sus organizaciones.

Las 10 competencias seleccionadas constituyen el paquete mínimo que el programa deberá fortalecer, pero seguramente se identificarán otras competencias que deberán ser incorporadas. La formación de profesionales bajo estos criterios, podría constituirse en el inicio de un proceso, que evolucione a la implementación de un sistema de certificación de profesionales en SAN. Para la implementación de esa segunda etapa, es necesario que alguna universidad en el país se apropie del proceso, ya que es más factible para un centro de educación superior cumplir todos los requisitos que un programa de este tipo requiere.

Modalidad y plan de estudios propuesto: el diplomado se deberá desarrollar mediante un sistema modular semipresencial, con encuentros donde se llevarán a cabo clases magistrales, conferencias internacionales, discusiones, trabajos de grupo, resolución de estudios de caso, panel de expertos y giras de campo. Además, se desarrollará un intenso trabajo vía online, donde los facilitadores mantendrán un contacto permanente con los participantes. Los contenidos del plan de estudios están divididos en siete módulos (Cuadro 7) que abarcan la mayoría de temas relacionados con la SAN y que son imprescindibles para un profesional que trabaja en programas y proyectos de este tipo. Los módulos serán facilitados por profesionales con experiencia en cada uno de ellos. Además de los módulos, durante el transcurso del diplomado se llevarán a cabo dos giras de campo para conocer experiencias reales de programas y proyectos de apoyo a la SAN. 
Cuadro 6. Competencias a fortalecer durante el proceso de formación en Seguridad Alimentaria y Nutricional.

\begin{tabular}{|c|c|c|}
\hline Competencias & Objetivos & Metodología de fortalecimiento \\
\hline \multicolumn{3}{|l|}{ Técnicas } \\
\hline Trabajo en equipo & $\begin{array}{l}\text { Entender las bondades del } \\
\text { trabajo en equipo y manejar } \\
\text { herramientas para su } \\
\text { aplicación. }\end{array}$ & $\begin{array}{l}\text { Discusiones de grupo, trabajos grupales, } \\
\text { presentación de informes conjuntos. }\end{array}$ \\
\hline $\begin{array}{l}\text { Resolución de } \\
\text { problemas }\end{array}$ & $\begin{array}{l}\text { Comprender y aplicar las fases } \\
\text { en la resolución de un } \\
\text { problema y en la toma de } \\
\text { decisiones asociadas al mismo. }\end{array}$ & $\begin{array}{l}\text { Resolución de estudios de caso, análisis de } \\
\text { alternativas de solución, priorización de acciones }\end{array}$ \\
\hline Comunicación & $\begin{array}{l}\text { Mejorar las habilidades } \\
\text { comunicativas, especialmente } \\
\text { las relacionadas con la } \\
\text { expresión en público. }\end{array}$ & $\begin{array}{l}\text { Presentaciones orales y escritas de trabajos } \\
\text { individuales y grupales }\end{array}$ \\
\hline $\begin{array}{l}\text { Información y } \\
\text { documentación }\end{array}$ & $\begin{array}{l}\text { Desarrollar el hábito de } \\
\text { investigación y documentación } \\
\text { ante una situación determinada }\end{array}$ & $\begin{array}{l}\text { Investigación bibliográfica de temas de interés, } \\
\text { consultas a expertos y presentación de resúmenes } \\
\text { ejecutivos }\end{array}$ \\
\hline \multicolumn{3}{|c|}{ (1) } \\
\hline $\begin{array}{l}\text { Orientación a } \\
\text { proyectos }\end{array}$ & $\begin{array}{l}\text { Comprender las fases de un } \\
\text { proyecto y los elementos a } \\
\text { considerar en su diseño e } \\
\text { implementación. }\end{array}$ & $\begin{array}{l}\text { Análisis de documentos de proyectos y visita a } \\
\text { proyectos en campo, discusión sobre teoría y } \\
\text { práctica. }\end{array}$ \\
\hline $\begin{array}{l}\text { Relaciones con } \\
\text { organizaciones }\end{array}$ & $\begin{array}{l}\text { Entender la importancia de } \\
\text { mantener buenas relaciones } \\
\text { con la diversidad de actores } \\
\text { que interactúan en un } \\
\text { territorio. }\end{array}$ & $\begin{array}{l}\text { Se fomentará el establecimiento de relaciones } \\
\text { institucionales duraderas entre los participantes. }\end{array}$ \\
\hline $\begin{array}{l}\text { Uso de nuevas } \\
\text { tecnologías }\end{array}$ & $\begin{array}{l}\text { Conocer y usar las nuevas } \\
\text { tecnologías de información y } \\
\text { comunicación. }\end{array}$ & $\begin{array}{l}\text { Uso de herramientas informáticas en discusiones } \\
\text { de grupo y consultas con facilitadores de módulos. }\end{array}$ \\
\hline \multicolumn{3}{|l|}{ Comportamiento } \\
\hline Liderazgo & $\begin{array}{lll}\text { Promover } & \text { actitudes } & \text { de } \\
\text { liderazgo y comprender } & \text { su } \\
\text { importancia en el marco } & \text { de } \\
\text { proyectos de desarrollo. } & \end{array}$ & $\begin{array}{l}\text { Toma de decisiones sobre aspectos relevantes del } \\
\text { diplomado y sobre acciones de seguimiento. }\end{array}$ \\
\hline Actitud abierta & $\begin{array}{l}\text { Desarrollar la actitud positiva } \\
\text { ante la crítica y la capacidad } \\
\text { de considerar la diversidad de } \\
\text { criterios que surgen alrededor } \\
\text { de un determinado tema. }\end{array}$ & Desarrollo de ejercicios de crítica y autocritica. \\
\hline Creatividad & $\begin{array}{l}\text { Fomentar el planteamiento de } \\
\text { ideas novedosas para la } \\
\text { solución de situaciones } \\
\text { problemáticas. }\end{array}$ & $\begin{array}{l}\text { Se crearán espacios para que los participantes } \\
\text { tomen sus propias decisiones en base a sus } \\
\text { intereses particulares. }\end{array}$ \\
\hline
\end{tabular}


Cuadro 7. Módulos a desarrollar en el Diplomado en Seguridad Alimentaria y Nutricional (SAN).

\begin{tabular}{cl}
\hline Módulo & \multicolumn{1}{c}{ Tema } \\
\hline 1 & $\begin{array}{l}\text { Conceptos generales sobre pobreza, } \\
\text { desarrollo humano y SAN }\end{array}$ \\
\hline 2 & $\begin{array}{l}\text { Situación actual y causas de la } \\
\text { inseguridad alimentaria a nivel mundial, } \\
\text { regional y local }\end{array}$ \\
\hline 3 & $\begin{array}{l}\text { Impactos de la inseguridad alimentaria y } \\
\text { propuestas de solución a la situación } \\
\text { actual }\end{array}$ \\
\hline 5 & $\begin{array}{l}\text { Institucionalidad y políticas de apoyo a } \\
\text { la SAN }\end{array}$ \\
\hline 5 & $\begin{array}{l}\text { Planificación de proyectos de apoyo a la } \\
\text { SAN }\end{array}$ \\
\hline 7 & $\begin{array}{l}\text { Educación para mejorar la SAN } \\
\text { evaluación final de trabajos individuales y }\end{array}$ \\
\hline
\end{tabular}

Evaluación de los participantes y del diplomado: la evaluación de conocimientos, habilidades y competencias adquiridas será un proceso continuo durante el desarrollo del diplomado. Una de las metodologías empleadas será consultar las organizaciones donde trabajan los participantes para determinar la adquisición de conocimientos y los cambios de actitud en cada uno de ellos.

Los módulos serán evaluados por los facilitadores con los participantes. En la evaluación de los módulos se considerarán los trabajos individuales y grupales, la participación en discusiones (presenciales y en línea) y la presentación de informes. Otro elemento que tendrá un valor en la evaluación final del diplomado es el trabajo individual de investigación, que será entregado y defendido en el último encuentro y consistirá en satisfacer una demanda de información para la toma de decisiones en sus organizaciones. También se brindará a los participantes la oportunidad de evaluar la organización y desarrollo del diplomado, de tal forma que sus comentarios y sugerencias sean la base para proponer mejora en futuras ediciones.

Durante el desarrollo del Diplomado y después de transcurrido el mismo, se establecerán contactos con las organizaciones donde trabajan los participantes para conocer su percepción sobre los impactos del diplomado en el desarrollo profesional de cada uno. Tres meses después de finalizado el último modulo, se elaborará un cuestionario que será enviado a los jefes inmediatos de cada participante y en donde mediante un serie de criterios se intentará determinar el nivel de aplicación de cada una de las competencias fortalecidas durante el proceso de formación.

\section{Bibliografía}

AEIPRO (Asociación Española de Ingeniería de Proyectos); IPMA (International Project Management Association). 2006. Bases para la competencia en dirección de proyectos versión 3.0. Trad. JM Almela. 5 ed. Valencia, España, Editorial Universidad Politécnica de Valencia. $237 \mathrm{p}$.

Ardón, C; A, Afonso. 2009. Percepciones sobre programas de formación e institucionalidad en Seguridad Alimentaria y Nutricional (SAN) en Honduras. Ceiba 50(1):21-39

Argüelles, A; Gonczi, A. 2001. Educación y capacitación basada en normas de competencia. Una perspectiva internacional. MX, Editorial Limusa. 315 p.

Barillas, M. 2004. Informe final I curso de postgrado en seguridad alimentaria y pobreza. Guatemala. $26 \mathrm{p}$.

Brígido, R. 2008. Certificación y normalización de competencias. Orígenes, conceptos y prácticas. Boletín No. 152 del Centro Interamericano para el Desarrollo del Conocimiento en la Formación Profesional (CINTERFOR). $24 \mathrm{p}$.

Carrión, R. 2003. Prospectiva, pertinencia y calidad de la educación universitaria. Nota científica. Industrial Data 6(1):103-105.

CSUCA (Consejo Superior de Universidades de Centroamérica); INCAP (Instituto de Nutrición de Centroamérica y Panamá); FAO. 2004. Plan de acción universitario (PAU) para la promoción de la seguridad alimentaria y nutricional en Centroamérica 2005-2006 Guatemala. 5 p.

FAO. 1996. Declaración de Roma sobre la seguridad alimentaria y nutricional. Roma, Italia. $4 \mathrm{p}$.

FAO; Universidad Rafael Landivar; FLACSO; FAUSAC; Fundacion Soros. 2006. Informe final II curso sobre seguridad alimentaria y pobreza. Guatemala. $67 \mathrm{p}$. 
Ardón y Afonso Gallegos: Formación Profesional Basada en Competencias como Estrategia de Apoyo

FAO. 2010. The state of food insecurity in the world. Addressing food insecurity in protracted crises. Economic and Social Development Department Food and Agriculture Organization of the United Nations. Rome, Italia.

Guerrero, D; I, de los Rios; F, Gómez; J, Guillen. 2010. Modelos internacionales de certificación de competencias profesionales: una caracterización de ocho modelos. Universidad Politécnica de Madrid, España. 23 p.

AENOR (Asociación Española de Normalización y Certificación). 2003. Evaluación de la conformidad. Requisitos generales para los organismos que realizan la certificación de personas ISO/EIC 17024: 2003. Madrid, España. 19 p.

Juliá, J. 2007. Rector de la Universidad Politécnica de Valencia. "La misión de la Universidad ya no es solamente formar profesionales" Entrevista a rector de la Universidad Politécnica de Valencia (en línea). Consultada 23 septiembre de 2011. Disponible en www.Ibercampus.es/articulos.asp?idarticulo=5197.

Lester, R.K. 2005. Universities, Innovation, and the Competitiveness of Local Economies: summary report from the local innovation project - phase I. Industrial Performance Center. Massachussets Institute of Technology. IPC Working Paper Series 05-010. 36p.

Mertens, L. 2000. La gestión por competencia laboral en la empresa y la formación profesional. Programa de Cooperación Iberoamericana para el Diseño de la Formación Profesional, Cumbre Iberoamericana. Madrid, España. 103 p.

Nonaka, I; Takeuchi, H. 1995. The Knowledge-Creating Company. New York, US. Oxford University Press.

Oseguera, M. 2005. Diagnóstico sobre políticas de investigación en las universidades. El caso de Honduras. Tegucigalpa, Honduras. 65 p.

PNUD. 2010. Objetivos de desarrollo del milenio Honduras 2010. Tercer informe de País. Tegucigalpa, Honduras. $254 \mathrm{p}$.
Plata M,L. 2003. La pertinencia de la educación superior. Elementos para su comprensión. Revista de la Educación Superior. XXXII (3), Núm. 127.

Quiñones, C. y Vélez, A. 2004. Algunas condiciones pedagógicas para la formación y el desarrollo de la investigación en la universidad. Revista Electrónica Actualidades Investigativas en Educación, Universidad de Costa Rica 4(001):1-27.

Rodríguez, E. 2009. El discurso de la formación basada en competencias profesionales. Un análisis crítico de la formación inicial de profesionales en la Educación Superior. REIFOP 1 (1):151-160.

Serón, A. 1999. El enfoque de las competencias profesionales: una solución conflictiva a la relación entre formación y empleo. Revista Complutense de Educación 10(1):335-360.

Tobón, S. 2008. La formación basada en competencias en la educación superior. El enfoque complejo. Universidad Autónoma de Guadalajara, Curso IGLU 2008. 30 p.

UNAH. 2008. Oferta educativa de los centros de educación superior 2008. Dirección de Educación Superior. (en línea). Consultada el 12 enero 2012. Disponible en https://www.unah.edu.hn/?cat=1252\&fcats

UNCR (Universidad Nacional de Costa Rica); UCR (Universidad de Costa Rica). 2009. Programa de maestría en gerencia en seguridad alimentaria y nutricional. $9 \mathrm{p}$.

Universidad Nacional Autónoma de Nicaragua-León; UCA (Universidad Centroamericana); UNA (Universidad Nacional Agraria). 2006. Programa de Estudio de Postgrado en Pobreza y Seguridad Alimentaria y Nutricional. 9 p.

WFP. 2011. Monitoring report on food security and nutrition Honduras. Fourth edition: April 2011. 12 p.

Recibido para publicación el 29 de octubre de 2011. Aceptado para publicación el 20 de febrero de 2012. 